\title{
von Willebrand Factor Mutation Enhancing Interaction with Platelets in Patients with Normal Multimeric Structure
}

\author{
Lars Holmberg, ${ }^{*}$ Judith A. Dent, ${ }^{\star}$ Reinhard Schneppenheim, ${ }^{\star}$ Ulrich Budde, ${ }^{5}$ Jerry Ware, ${ }^{*}$ and Zaverio M. Ruggeri ${ }^{*}$ \\ ${ }^{*}$ Roon Research Laboratory for Arteriosclerosis and Thrombosis, Division of Experimental Hemostasis and Thrombosis, Department of \\ Molecular and Experimental Medicine and Committee on Vascular Biology, The Scripps Research Institute, La Jolla, California 92037; \\ ${ }^{\ddagger}$ Kinderklinik der Christian-Albrechts-Universität zu Kiel, 2300 Kiel 1, Germany; and ${ }^{\S}$ Blutspendedienst des AK Harburg, D2100 \\ Hamburg, Germany
}

\begin{abstract}
Variant von Willebrand disease designated as type I New York or type Malmö is characterized by enhanced ristocetin-induced platelet agglutination with normal von Willebrand factor multimeric distribution in plasma. We have studied four such patients belonging to three unrelated families and found in all of them a unique cytosine-to-thymine transition changing the codon for Pro ${ }^{503}$ (CCG) to Leu (CTG). In three patients the mutant allele also had a silent mutation in the codon for Ser ${ }^{500}$ (TCG $\rightarrow$ TCA ). Both nucleotide changes are present in the von Willebrand factor pseudogene; however, the characterization of distinctive markers where the gene and pseudogene differ, as well as the examination of amplified cDNA derived from platelet mRNA, confirmed that the abnormality occurs in the von Willebrand factor gene of the patients. Moreover, recombinant expression of the isolated glycoprotein Ib-binding domain of von Willebrand factor provided direct evidence that the Pro $^{503} \rightarrow$ Leu mutation is responsible for enhanced platelet reactivity to lower ristocetin concentrations. These results define a new structural element affecting the affinity of von Willebrand factor for glycoprotein Ib and establish the molecular basis of a variant form of von Willebrand disease. (J. Clin. Invest. 1993. 91:2169-2177.) Key words: bleeding • mutation • pseudogene $\bullet$ thrombosis $\bullet$ von Willebrand disease
\end{abstract}

\section{Introduction}

von Willebrand disease (1) is a common inheritable bleeding disorder characterized by heterogeneous phenotypic manifestations and caused by quantitative or qualitative defects of vWF, a glycoprotein with typical multimeric structure (2). By convention, the disease is classified into three categories: type I, with moderately decreased plasma concentration of a seemingly normal vWF; type II, with qualitatively abnormal vWF at normal or near normal plasma concentration; and type III, with very low or undetectable vWF and, unlike types I and II, recessive inheritance. A number of subtypes exist within these

Dr. Holmberg's present address is Department of Paediatrics, University of Lund, University Hospital, S-221 85 Lund, Sweden.

Address reprint requests to Dr. Zaverio M. Ruggeri, The Scripps Research Institute, SBR-8, 10666 North Torrey Pines Road, La Jolla, CA 92037.

Received for publication 24 July 1992 and in revised form 9 December 1992.

J. Clin. Invest.

(c) The American Society for Clinical Investigation, Inc.

0021-9738/93/05/2169/09 \$2.00

Volume 91, May 1993, 2169-2177 categories (1). Type II variants have attracted special interest because elucidation of the underlying molecular abnormalities may yield insights into vWF structure-function relationships $(3,4)$.

vWF interacts with components of the vessel wall and with the platelet GP complexes Ib-IX-V and IIb-IIIa to promote platelet adhesion and thrombus formation at sites of vascular injury (2). In that normal vWF in solution has no measurable interaction with platelets, the initiation of vWF-dependent platelet thrombus formation is thought to require conformational changes in vWF bound to the exposed subendothelium and / or subjected to the effect of shear forces, resulting in highaffinity binding to GP Ib. Thus, different conformations of a specific functional domain may regulate the interaction of vWF with GP Ib and its role in hemostasis (2).

In the type IIB variant of von Willebrand disease, vWF in solution displays enhanced affinity for GP Ib, as shown either directly with the purified protein (5) or by the induction of aggregation in platelet-rich plasma at ristocetin concentrations lower than those effective in normal samples (6). The affected patients also exhibit absence of the largest vWF multimers in plasma, probably owing to their spontaneous binding to circulating platelets (7). In some cases, this interaction may induce in vivo platelet aggregation and thrombocytopenia $(8,9)$, which always occur in type IIB von Willebrand disease after administration of 1-deamino-8-D-arginine vasopressin (DDAVP) $^{1}(10)$. Different gene mutations have been identified in a number of these patients $(3,11-13)$ and all have been found to be localized in a domain of vWF, the disulfide loop created by the linkage of $\mathrm{Cys}^{509}$ and Cys ${ }^{695}$, that has been indicated as an important element in the regulation of vWF binding to GP Ib $(2,3)$.

Besides type IIB - and platelet-type $(14,15)$ or pseudo-von Willebrand disease (16) which are, however, GP Ib defects (17) -there is another form of von Willebrand disease in which platelet aggregation and vWF binding to platelets occur at lower ristocetin concentrations than in normal subjects. In contrast to type IIB, however, there is no spontaneous aggregation nor thrombocytopenia even after DDAVP administration. Furthermore, the full range of vWF multimers is present in the plasma of the patients as is characteristic of type I von Willebrand disease. The latter observation suggested the designation "type I New York" for this variant (18), but patients with the same phenotype were considered by others as belonging to type II (type Malmö) because of their functional vWF abnormality (19). We have now investigated four patients from three unrelated families with this form of the disease and

1. Abbreviations used in this paper: DDAVP, deamino-8-D-arginine vasopressin; DEPC, diethylpyrocarbonate. 
here demonstrate in all of them the same gene mutation leading to an amino acid substitution at position 503 of the mature vWF subunit. This abnormality has been reproduced by in vitro mutagenesis and the properties of the expressed protein confirm the relationship between the mutation and enhanced interaction with platelets in the presence of ristocetin.

\section{Methods}

Blood collection and functional studies of hemostasis. Blood was collected with trisodium citrate as anticoagulant $(0.012 \mathrm{M}$ final concentration); platelet-rich plasma and platelet-poor plasma were prepared as described previously (19). Factor VIII procoagulant activity, von Willebrand factor antigen, and ristocetin-cofactor activity were measured using methods previously described (19). The multimeric structure of plasma vWF was evaluated in agarose gels containing SDS, as reported (20). Bleeding time was measured using a template method (19). Ristocetin-induced platelet aggregation was evaluated by measuring either the extent or the maximal initial velocity of aggregation upon addition of varying final concentrations of ristocetin $(6,7,21)$. Normal reference values for each assay were obtained in the normal population of the two centers where the clinical studies were performed (Malmö and Hamburg). Control subjects were from the laboratory staff. All individuals that participated in the study were aware of its experimental nature and gave their informed consent, according to the Declaration of Helsinki.

Nucleic acid analyses. DNA was extracted from whole blood or buffy coat using standard procedures (22). Platelet RNA was prepared from washed platelets obtained from $50 \mathrm{ml}$ of blood (23). The platelets were lysed in a solution composed of $4 \mathrm{M}$ guanidinium isothiocyanate (Gibco-BRL, Gaithersburg, MD), $5 \mathrm{mM}$ sodium citrate, $100 \mathrm{mM} 2$ mercaptoethanol, $0.5 \% \mathrm{~N}$-laurylsarcosine, and $0.1 \%$ antifoam $\mathrm{A}$ in diethylpyrocarbonate (DEPC)-treated water. $3 \mathrm{ml}$ of platelet lysate were centrifuged through a CsCl cushion for $20 \mathrm{~h}$ at $35,000 \mathrm{rpm}, 20^{\circ} \mathrm{C}$, in a model SW 50.1 rotor $(150,000 \mathrm{~g}$; Beckman Instruments, Inc., Fullerton, CA). The RNA pellet was dissolved in $20 \mu \mathrm{l}$ of DEPCtreated water and $1 \mu$ l of ribonuclease inhibitor (Promega Corp., Madison, WI). The RNA was stored at $-70^{\circ} \mathrm{C}$ as an ethanol precipitate.

The portion of vWF gene (24) coding for residues 463-736 was amplified from genomic DNA using the PCR (25) and the previously described oligonucleotide primers 226 and 227A (26). These primers selectively amplify a portion of exon 28 without amplifying the homologous sequence within the vWF pseudogene due to $3^{\prime}$ basepair mismatches between the oligonucleotides and the pseudogene (26). After amplification the fragments were cloned in the filamentous phage M13 for DNA sequence analysis.

Single stranded $\mathrm{vWF} \mathrm{cDNA}$ fragments were generated from platelet RNA using Moloney murine leukemia virus reverse transcriptase primed by a vWF-specific oligonucleotide (complementary to the vWF mRNA coding for residues 899-908). Aliquots of this first reaction were subjected to a PCR using oligonucleotides that amplify a vWF fragment representing the coding sequence for residues 441-681. The oligonucleotides used in the PCR correspond to coding sequence from nucleotide $23 / 196$ to $23 / 220$ within exon 27 and to noncoding sequence complementary to nucleotides $24 / 841$ to 24 / 864 within exon 28 (nucleotide numbers are from reference 24 ). Restriction enzyme digestion with DdeI was performed on the amplified fragments using the supplier's recommended digestion conditions.

In vitro mutagenesis and expression of recombinant $v W F$ fragments. The Pro ${ }^{503} \rightarrow$ Leu amino acid substitution was expressed utilizing a previously described construct, pAD5/WT (27), that directs the synthesis in Chinese hamster ovary ( $\mathrm{CHO}$ ) cells of a $116-\mathrm{kD}$ homodimeric fragment of vWF, referred to as $r 116$. The expressed fragment contains residues 441-733 of mature vWF and has been shown to recapitulate the properties of native $\mathrm{vWF}$ with regard to its interaction with platelet GP Ib (27). The normal vWF coding sequence within $\mathrm{pAD} / \mathrm{WT}$ was cloned into $\mathrm{pBS} / \mathrm{KS}^{-}$(Stratagene, Inc., La Jolla) and a uracil-containing single stranded template was generated using the dut $^{-}$ung $^{-}$Escherichia coli strain CJ236 (28). After mutagenesis and verification of the entire vWF coding sequence, the vWF insert was subcloned into the Xhol/Notl site of the expression plasmid pCDM ${ }^{\text {neo }}$ as previously described (27). The mutant plasmid contains the same coding sequence as pAD5/WT with two differences: the Pro ${ }^{503} \rightarrow$ Leu codon substitution, as seen in the patients, and a change in the codon for $\operatorname{Asp}^{498}$ (GAC $\rightarrow$ GAT), inserting a new EcoRV site used for the screening of mutant plasmids.

Cell culture, DNA transfection, and selection of stable transformants. $\mathrm{CHO}$ cells were maintained in $5 \% \mathrm{CO}_{2}$ and grown in DME supplemented with $10 \%$ fetal calf serum, $0.5 \mathrm{mM}$ nonessential amino acids, and $2 \mathrm{mM}$ L-glutamine (Whittaker Bioproducts, Walkersville, MD). Cells were cultured at a density of $1.5 \times 10^{5}$ in $60-\mathrm{mm}$ dishes and transfection mediated by calcium phosphate was achieved with $10 \mu \mathrm{g}$ of DNA per dish, as described previously $(27,29)$. Stable transformants were established by growing cells for $10-14 \mathrm{~d}$ in the presence of $0.8 \mathrm{mg} / \mathrm{ml}$ of the antibiotic G418 (Sigma Chemical Co., St. Louis, $\mathrm{MO}$ ) and isolated colonies were expanded in 12-well plates. The culture supernatants were examined for the presence of vWF antigen by spotting 200- $\mu$ laliquots onto nitrocellulose membranes and then incubating the membranes sequentially with anti-vWF monoclonal antibodies and ${ }^{125} \mathrm{I}$-labeled rabbit anti-mouse $\mathrm{IgG}$, followed by autoradiographic detection (3).

Characterization of expressed $v W F$ fragments. Serum-free culture media were concentrated by centrifugation using a Centricon 30 (Amicon, Beverly, MA). Quantitative determination of vWF antigen in the concentrated media was performed with Laurell electroimmunoassay $(30,31)$, using a pool of monoclonal antibodies recognizing epitopes within vWF residues 449-728. Affinity-purified $r 116 \mathrm{vWF}$ fragment was used as a reference standard for this assay.

Western blot analysis of the $r 116$ fragment was performed with two anti-vWF monoclonal antibodies: NMC-4, recognizing a disulfide-dependent conformation of vWF conferred by the Cys ${ }^{509}$-Cys ${ }^{695}$ bond and retained in the isolated GP Ib-binding domain $(32,33)$, and $\mathrm{LJ}$ RG46, reacting with a conformation-independent linear epitope located within residues $474-488$ of the mature vWF subunit (32). Immunoreactive proteins were visualized by subsequent incubation with ${ }^{125} \mathrm{I}$ labeled rabbit anti-mouse IgG followed by autoradiography.

Immunopurification of normal and mutant $r 116 \mathrm{vWF}$ fragments was performed by applying $500 \mathrm{ml}$ of culture supernatant onto a $20-\mathrm{ml}$ column prepared by coupling the monoclonal antibody NMC-4 to $\mathrm{CNBr}$-activated Sepharose as previously described (27). Protein determination was performed with bicinchonic acid assay (Pierce Chemical Co., Rockford, IL).

For aggregation studies, either immunopurified fragments or concentrated culture media were used. The latter were obtained by filtering $100 \mathrm{ml}$ of serum-free culture supernatant through $0.45-\mu \mathrm{m}$ Nalgene filters, then dialyzing extensively against a buffer composed of $20 \mathrm{mM}$

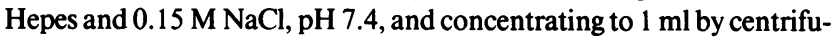
gation using a Centricon 30 filter. Aggregation was evaluated using human washed platelets prepared by the method of Walsh et al. (34) with modifications described elsewhere (35); the final platelet count in the assay was $1.5 \times 10^{8} / \mathrm{ml}$. A siliconized glass cuvette containing the experimental mixture was placed in an aggregometer (Chrono-Log Corp., Havertown, PA) at $37^{\circ} \mathrm{C}$ with constant stirring (1,200 rpm); aggregation was induced by the addition of ristocetin and monitored through the increase in light transmission.

\section{Results}

Characterization of the patients studied. The four patients described in this study are from three families, one from Sweden, one from Germany, and one from the UK (Bermuda). The Swedish family is the original one in which the occurrence of this variant form of von Willebrand disease was described in eight affected members. The patient examined here, patient 1 , 
Table I. Laboratory Data of the Four Patients Studied

\begin{tabular}{|c|c|c|c|c|c|c|c|}
\hline & Sex & Age & $\begin{array}{l}\text { Bleeding } \\
\text { time }\end{array}$ & FVIII:C & vWF:Ag & RiCof & RIPA \\
\hline & & $y r$ & $\min$ & & $U / d l$ & & $\mathrm{mg} / \mathrm{ml}$ \\
\hline Patient 1 & $\mathbf{M}$ & 20 & 14 & 57 & 55 & 46 & 0.90 \\
\hline Normal & & & $<12$ & $60-160$ & $50-175$ & $50-160$ & $>1.0$ \\
\hline Patient 2 & $\mathrm{~F}$ & 54 & 11 & 50 & 87 & 64 & 0.4 \\
\hline Patient 3 & $\mathrm{~F}$ & 27 & - & 41 & 110 & 78 & 0.5 \\
\hline Patient 4 & $\mathrm{~F}$ & 38 & 5 & 73 & 68 & 84 & 0.5 \\
\hline Normal & & & $<9$ & $60-160$ & $50-160$ & $50-160$ & $>0.6$ \\
\hline
\end{tabular}

Factor VIII procoagulant activity (FVIII:C), von Willebrand factor antigen (vWF:Ag), and ristocetin cofactor activity (RiCof) are expressed as arbitrary units per deciliter; average normal plasma contains $100 \mathrm{U} / \mathrm{dl}$. Note that the bleeding time was not tested in patient 3 . Ristocetin-induced platelet aggregation (RIPA) was evaluated with two different methods: in patient 1, by determining the concentration of ristocetin inducing aggregation with an initial velocity of at least $20 \mathrm{~mm} / \mathrm{min}$; in patients 2,3 , and 4, by determining the concentration of ristocetin inducing aggregation with at least $50 \%$ maximal response. Different normal reference values are reported for the patients studied in two different centers (Malmö for patient 1; Hamburg for patients 2, 3, and 4).

reported previously as III: 1 (19), is the son of the original propositus (deceased) and was diagnosed in the course of family studies; he had no obvious bleeding history. The German family has five affected members and the inheritance is autosomal dominant as in the Swedish family; the mother and daughter, patients 2 and 3, are characterized in this report. Patient 2 had bleeding considered abnormal after two tooth extractions and suffered from cutaneous hematoma and menorrhagia; patient 3 had no obvious bleeding history. The female patient from Bermuda, patient 4 , was the only member of her family with an increased bleeding tendency; she experienced abnormal blood loss after tooth extractions, as well as cutaneous hematoma and increased blood loss after an abortion. Altogether, the symptoms in all affected patients, when present, were considered mild.

The relevant laboratory findings are summarized in Table I; of note, the bleeding time ranged from normal (patient 4 ) to only moderately prolonged. In all the patients, platelets in platelet-rich plasma were aggregated at concentrations of ristocetin lower than those necessary to cause aggregation in normal samples, a finding representing the distinctive phenotypic feature of these individuals $(18,19)$. Plasma vWF:Ag levels were normal in all cases, and ristocetin cofactor activity (which is measured at nonlimiting ristocetin concentrations) was moderately decreased only in patient 1. FVIII:C was normal in patient 4 but was moderately decreased in the others; moreover, the ratio between plasma FVIII:C and vWF:Ag was low $(<0.6)$ in patients 2 and 3, belonging to the same family. For practical reasons, these assays of FVIII:C were performed on frozen samples; this may account for the discrepancies observed due to the labile nature of factor VIII activity. All vWF multimers were present in plasma, with a normal relative proportion of species of different molecular mass; thus, the multimeric structure of patient vWF was indistinguishable from that of normal controls (Fig. 1). No patient had thrombocytopenia; moreover, DDAVP administration to patient 2 shortened the bleeding time, raised factor VIII and vWF levels but, unlike in type IIB von Willebrand disease (10), did not induce thrombocytopenia.

Genetic studies. DNA sequence analysis was performed on the vWF gene of the four patients under consideration. Because their phenotypic abnormality was characterized by enhanced
vWF interaction with platelets in the presence of ristocetin, we focused our attention on the GP Ib-binding domain of vWF $(32,35)$. The sequence encoding residues $449-673$ of the mature vWF subunit was determined after cloning PCR-generated fragments into the filamentous phage M13. The analysis of eight to twelve clones per DNA sample demonstrated that each patient had, in addition to a normal allele, a mutant allele in which a $\mathrm{C} \rightarrow \mathrm{T}$ transition (confirmed in at least two clones) changed the codon for amino acid residue 503 from the normal proline (CCG) to leucine (CTG) (Fig. 2). Furthermore, patients 2,3 , and 4 , but not patient 1 , had a $G \rightarrow$ A silent mutation in the codon for $\operatorname{Ser}^{500}$ ( TCG $\rightarrow$ TCA) that was linked to the allele containing the Pro ${ }^{503} \rightarrow$ Leu substitution (Fig. 2). The base change in the codon for $\operatorname{Ser}^{500}$ creates a new Ddel restriction site, the presence of which on a single allele was confirmed in the three patients (Fig. $3 \mathrm{~A}$ ).

Sequence analysis of the vWF gene based on PCR-generated fragments can be affected by interference from a highly homologous pseudogene possessing a structural organization similar to that of the "true" gene in the region containing the coding sequence of interest for these studies. The pseudogene has both TCA and CTG at positions homologous to those coding for amino acids 500 and 503 in the vWF gene, thus a sequence identical to that seen in the cloned DNA fragments from three patients. That the pseudogene did not influence our results, however, was clearly demonstrated by the identifica-

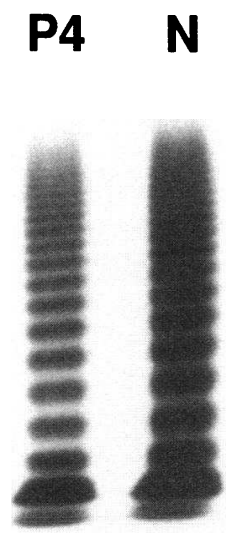

Figure 1. SDS-agarose gel electrophoresis of plasma vWF in patient $4(P 4)$ and a normal control $(N)$. Note that the distribution and the relative proportion of the different vWF multimers is identical in the patient sample as compared to normal. 

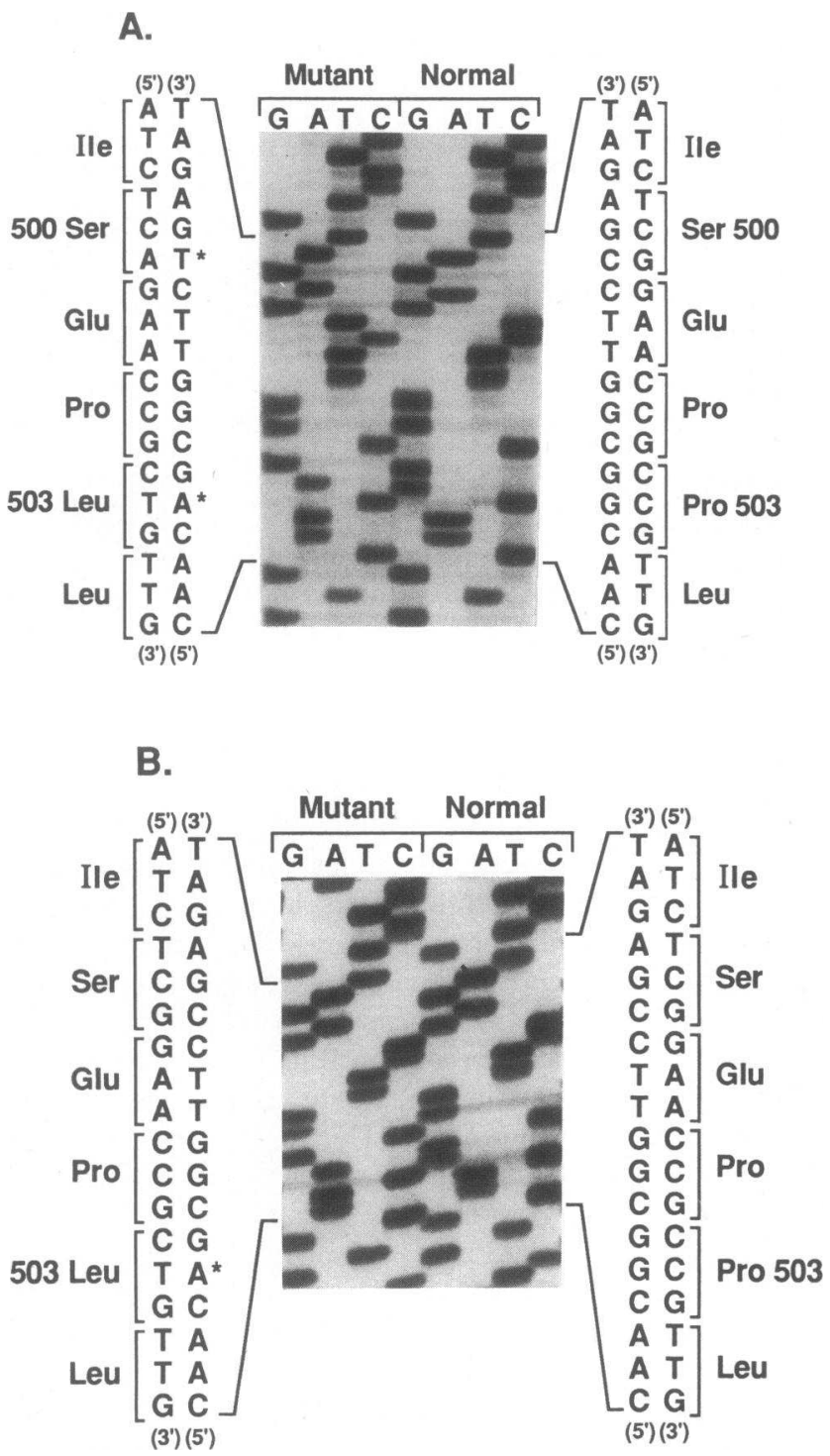

Figure 2. DNA sequence of normal and mutated vWF alleles. The lettering indicates the base sequence corresponding to the codons of amino acid residues 499-504 of the mature vWF subunit, as shown on the left and right. The figure presents the sequence of the noncoding strands with the coding strands aligned; the respective $3^{\prime}$ and $5^{\prime}$ ends are indicated. $(A)$ Patient 2, showing the mutation in the codon for residue 500 as well as the Pro ${ }^{503} \rightarrow$ Leu substitution; $(B)$ patient 1 , showing the normal codon for $\mathrm{Ser}^{500}$, but the same mutation of residue 503 as seen in patient 2 .

tion of the expected gene sequence in the mutant clones at all the 16 other positions where gene and pseudogene have been shown to diverge (26). Moreover, additional evidence that the observed mutations reflect vWF gene sequence in the patients was obtained by characterizing the platelet mRNA prepared from patient 2. A 746-bp fragment was amplified from the corresponding cDNA with two primers representing sequences in exons 27 and 28 separated by a 2-kb intron; thus, the absence of any larger fragment in the PCR product demonstrated the lack of genomic DNA contamination in the platelet cDNA preparation. The cDNA fragment was found to contain the DdeI restriction site corresponding to the silent mutation in the codon for $\operatorname{Ser}^{500}$ (Fig. $3 \mathrm{~B}$ ), providing evidence that the allele containing the linked Pro ${ }^{503}$ to Leu substitution is transcribed; the mutations, therefore, are truly present in the vWF gene.

Polymorphic markers of the vWF gene were then characterized to reveal the existence of possible unknown relationships between the three apparently unrelated families. Patient 1 , who lacked the silent mutation at position 500 , had the codon for aspartic acid 709 in the mutant allele, while the others had histidine, a reported vWF polymorphism (24). Patient 4 and patients 2 and 3 (mother and daughter) had concordant sequence in the mutant clones at the polymorphic positions 618 (36) and 709; however, further analysis of the vWF gene for restriction fragment length polymorphisms and variable number tandem repeats showed that both mutant and normal alleles from patient 4 differed from those in patients 2 and 3 (Table II).

Studies with expressed recombinant fragments. The mutation detected in the patients at position 503 was expressed in the homodimeric 116-kD fragment of vWF known to possess the GP Ib-binding properties of the intact molecule; the mutant fragment was designated $r 116$ (P503L). The dialyzed and concentrated supernatants from $r 116$ and $r 116$ (P503L) cultures contained 57 and $65 \mu \mathrm{g}$ of vWF fragment per milligram protein, respectively, as estimated from protein determination and electroimmunoassay of vWF with purified $r 116$ as standard. The immunoaffinity purified species had protein contents of $150 \mu \mathrm{g} / \mathrm{ml}$ for $r 116$ and $208 \mu \mathrm{g} / \mathrm{ml}$ for $r 116$ (P503L) after 20 -fold concentration of column eluates. Western blot demonstrated that the concentrated tissue culture supernatants and the purified preparations contained similar $116-\mathrm{kD}$ species reactive with the monoclonal antibody NMC-4 (Fig. 4). Thus, the Pro ${ }^{503} \rightarrow$ Leu substitution did not alter the ability of the expressed fragment to assemble into a dimeric species like the normal fragment. By using the conformation-independent antibody, LJ-RG46 (not shown), both $r 116$ and $r 116$ (P503L) gave a similar immunoreactive pattern, exhibiting the dimer of $116 \mathrm{kD}$ and some monomeric fragment of $52 / 48 \mathrm{kD}(3)$. This further demonstrated that the normal and mutant fragments were expressed in a comparable manner.

The concentrated tissue culture supernatants at a final vWF fragment concentration of $2.8 \mu \mathrm{g} / \mathrm{ml}$ ( $24 \mathrm{nM}$ calculated as $116-\mathrm{kD}$ dimer) supported the aggregation of platelets induced by ristocetin at $1.25 \mathrm{mg} / \mathrm{ml}$ final concentration. Dose-response curves with decreasing concentrations of ristocetin demonstrated that the fragment with normal sequence supported aggregation with as little as $0.4 \mathrm{mg} / \mathrm{ml}$ of ristocetin, albeit with diminished response, while $r$ 116(P503L) supported aggregation even at $0.15 \mathrm{mg} / \mathrm{ml}$ ristocetin (Fig. 5). Similar results were obtained with the immunoaffinity-purified fragments used at a final concentration of $60 \mathrm{nM}$ (calculated as $116-\mathrm{kD}$ dimer); $r 116$ supported aggregation at 0.4 but not $0.3 \mathrm{mg} / \mathrm{ml}$ of ristocetin, whereas $r 116(\mathrm{P} 503 \mathrm{~L})$ supported aggregation at $0.2 \mathrm{mg} / \mathrm{ml}$ of ristocetin (Fig. 6). Platelet aggregation could not be elicited in the absence of ristocetin even when the concentration of $r$ 116(P503L) was raised 10-fold.

\section{Discussion}

These studies demonstrate that the same missense mutation identified in three unrelated families in the sequence of mature vWF, Pro ${ }^{503} \rightarrow$ Leu, is the cause of the von Willebrand disease variant called type Malmö (19) and, presumably, of the phenotypically identical variant reported as type I New York (18). 
Patient
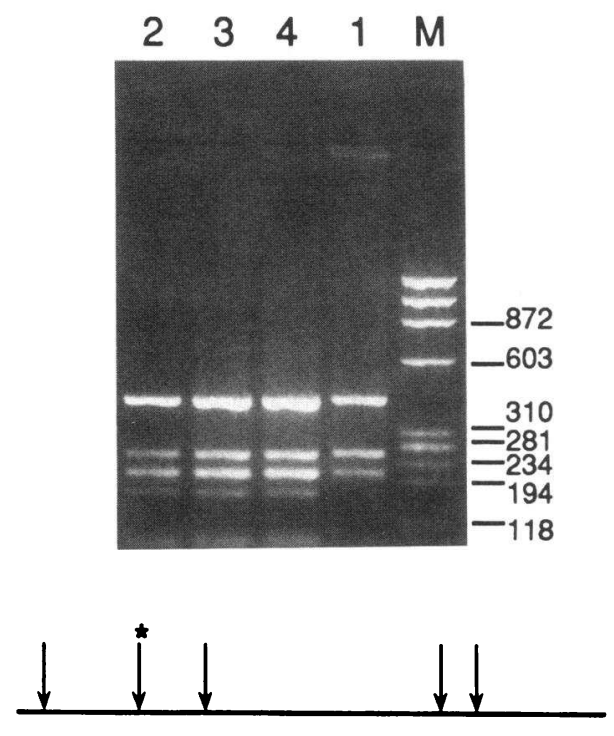

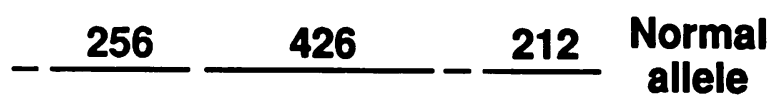

$-\frac{170}{86} \underset{426}{-212} \begin{gathered}\text { Mutant } \\ \text { allele }\end{gathered}$
B
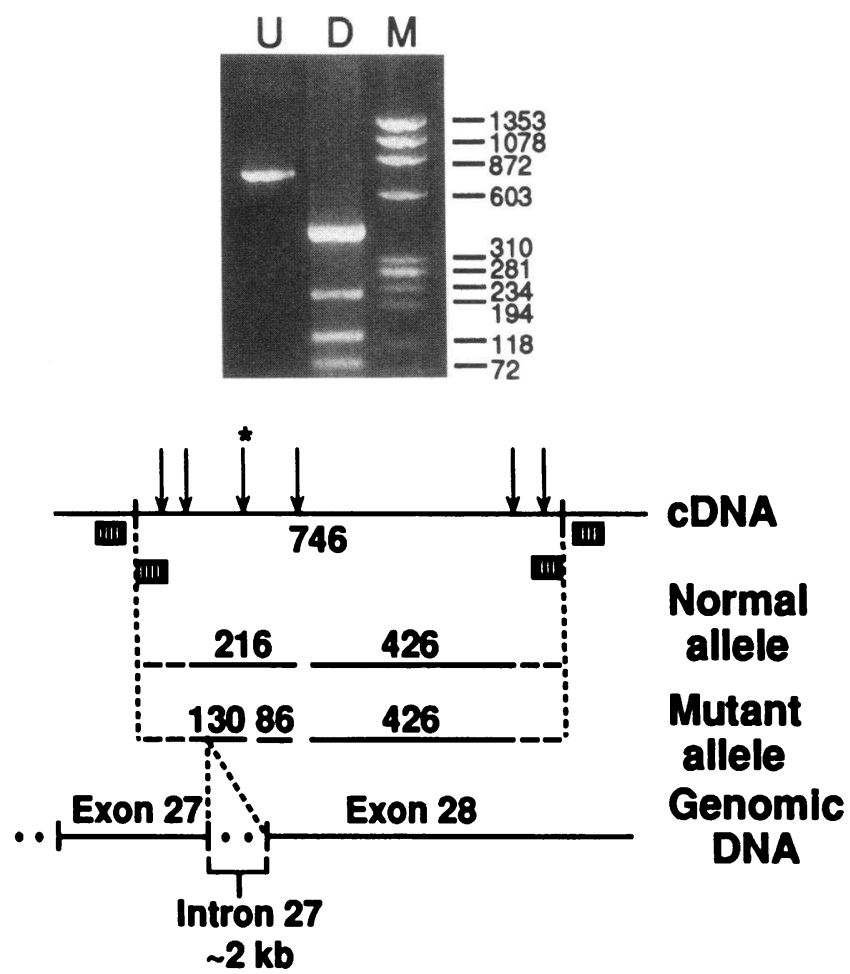

Figure 3. DdeI cleavage of PCR-amplified fragments demonstrating the TCG $\rightarrow$ TCA silent mutation in the codon for Ser ${ }^{500}$ of the mature vWF subunit. A continuous line represents the uncleaved fragments; arrows indicate cleavage sites and the asterisk indicates the site of mutation. Marker lanes $(M)$ contain a HaelII digest of $\Phi$ X174 RF DNA; the size of the corresponding fragments (bp) is shown on the right. ( $A$ ) Cleavage of a $958 \mathrm{bp}$ fragment amplified from genomic DNA with oligonucleotide primers described by Mancuso et al. (26). Unique fragments of 170 and $86 \mathrm{bp}$ are present in patients 2,3 , and 4 , but not in patient 1 , in addition to the normal fragments of 426,256 , and $212 \mathrm{bp}$. Two small fragments of 20 and $46 \mathrm{bp}$ migrate with the primers and are not visualized. (B) Cleavage of a 746-bp fragment amplified from vWF cDNA of patient 2 as described in Methods. $U$, uncleaved; $D$, DdeI-cleaved fragment. In the normal allele (not shown in this experiment) there are five cleavage sites, two corresponding to exon 27 and three to exon 28, as shown in the schematic representation of genomic DNA. The extra cleavage site at the codon for serine 500 in the mutant allele creates unique fragments of 130 and $86 \mathrm{bp}$, seen in the heterozygous patient in addition to the normal fragments of 426 and $216 \mathrm{bp}$. Four small fragments of 20-35 bp migrate with the oligonucleotide primers and are not visualized.

The mutation was detected in only one of the alleles, in agreement with the autosomal dominant mode of transmission of the bleeding disorder; moreover, in two of the three families (three out of four patients) the same allele exhibited a nucleotide substitution in the codon for serine 500. The two observed mutations create a sequence identical to that of the vWF pseu-

Table II. Polymorphisms Detected in the vWF Gene of Patients 2, 3, and 4

\begin{tabular}{cccc}
\hline Patient & TaqI(A) & XbaI & VNTR \\
\hline 2 & $-/-$ & $-/-$ & $10 / 13$ \\
3 & $-/-$ & $-/-$ & $12 / 13$ \\
4 & $+/+$ & $+/+$ & $7 / 7$
\end{tabular}

Polymorphisms were detected by restriction fragment length analysis after digestion of genomic DNA with TaqI (52) and XbaI (53), as indicated, and by determination of the variable number of tandem repeats (VNTR) (54). Patients 2 and 3 are mother and daughter. +, allele with polymorphic cleavage site; - , allele without it. dogene (26), thus raising the concern that the latter might have interfered in the cloning and sequencing procedures adopted to characterize the patients. That this was not the case, however, is demonstrated by the fact that the sequence of the mutant clones was found to be identical to that reported for the vWF gene at 16 other positions that are known to diverge from the pseudogene (26); and by the identification of a transcript from the mutant allele, as evidenced by analysis of cDNA generated from platelet mRNA.

The most characteristic feature of the von Willebrand disease variant described here is that aggregation is induced in platelet-rich plasma by lower ristocetin concentrations than necessary in normal subjects. Ristocetin-induced aggregation is known to involve the binding of vWF to platelet GP Ib (35) and, thus, is thought to mirror one of the crucial mechanisms supporting platelet adhesion and aggregation at high shear forces $(37,38)$. The GP Ib-binding site in vWF is located between residues 449 and 728 of the mature subunit $(32,39,40)$ and can be expressed in a native dimeric conformation as a fragment of $116 \mathrm{kD}$ secreted from $\mathrm{CHO}$ cells $(3,27)$. This fragment reproduces the properties of full-length vWF with regard to ristocetin-induced interaction with GP Ib, including 


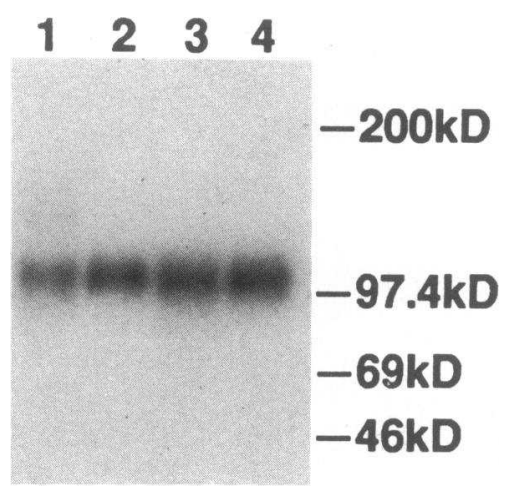

Figure 4. Western blot analysis of expressed recombinant fragments of vWF. Concentrated tissue culture supernatants and immunoaffinity-purified vWF fragments were electrophoresed in an SDS-polyacrylamide gel and reacted with the anti-vWF monoclonal antibody NMC-4 after transfer to a nitrocellulose membrane. The

electrophoretic mobility of known molecular mass markers is shown on the right. Lane 1, purified $r 116$; lane 2, purified $r 116$ (P503L); lane $3, r 116$ culture supernatant; lane $4, r 116(\mathrm{P} 503 \mathrm{~L})$ culture supernatant.

ristocetin-induced platelet aggregation. Although one cannot exclude a priori that the consequences of a single amino acid substitution, or other mutations, may be different in an isolated vWF fragment as opposed to the intact multimeric molecule, results documented previously with regard to a type IIB mutation (3) have shown that expression of the isolated 116$\mathrm{kD}$ fragment can reproduce typical phenotypic abnormalities of type IIB vWF. Thus, expression of this fragment was used to evaluate the functional consequences of the Pro ${ }^{503} \rightarrow$ Leu mutation detected in the patients described here. Indeed, the results obtained with the present studies lend further support to the validity of the approach based on isolated domain expression to study the functional consequences of mutations in the complex vWF molecule.

Platelet aggregation studies were performed using both tissue culture supernatants and immunopurified $\mathrm{vWF}$ fragments derived from them. With the former we could exclude that the results obtained were solely caused by effects of the purification procedure on the functional properties of the vWF fragments;

A

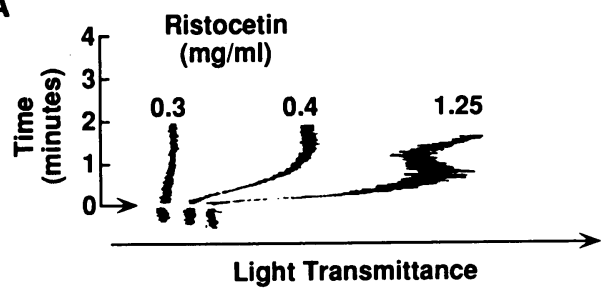

B

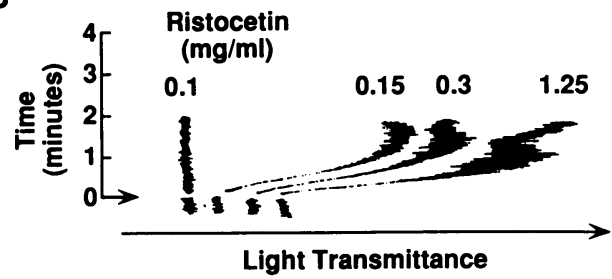

Figure 5. Platelet aggregation elicited by ristocetin in the presence of concentrated tissue culture supernatants. The numbers indicated for each trace represent the final ristocetin concentration $(\mathrm{mg} / \mathrm{ml})$ added to the platelet suspensions at the time indicated by an arrow. $(A)$ Normal $r 116$ fragment; (B) mutant $r 116$ (P503L) fragment.
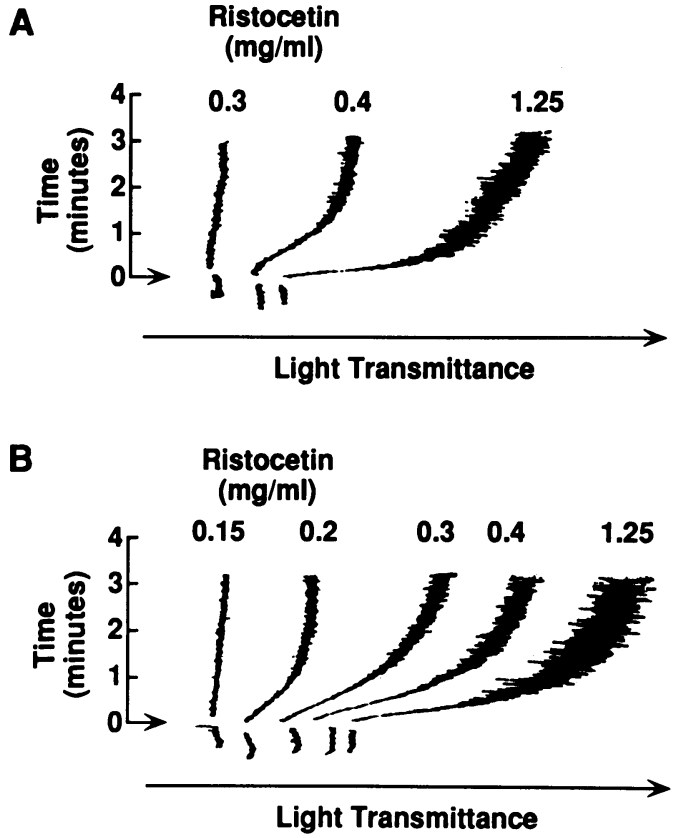

Figure 6. Platelet aggregation elicited by ristocetin in the presence of immunoaffinity purified vWF fragments. The presentation of results is the same as in Fig. 5. $(A)$ Normal $r 116$ fragment; $(B)$ mutant $r 116(\mathrm{P} 503 \mathrm{~L})$ fragment.

with the latter we could rule out the possibility that other secreted molecules present in the culture media interfered with the functional assay. Good aggregation response at $1.25 \mathrm{mg} / \mathrm{ml}$ ristocetin concentration required more purified than nonpurified vWF fragment, suggesting that the purification procedure may negatively affect the functional properties of the molecule. With either type of material, however, $r$ 116(P503L) supported platelet aggregation at lower ristocetin concentrations than $r 116$ with normal sequence, thus reproducing the typical phenotypic abnormality of the von Willebrand disease variant under consideration. The differences between the mutant and normal fragments were more pronounced than expected on the basis of the moderately increased ristocetin sensitivity in the patient platelet-rich plasma $(18,19)$. This may be explained by the fact that the patients have a mixture of normal and abnormal vWF subunits while the expressed mutant protein contained only abnormal species. In our experimental system utilizing washed platelets, aggregation was induced by low ristocetin concentrations that do not elicit aggregation in plasma; this reflects the fact that in the latter case ristocetin binds to other plasma proteins, thus decreasing its effective concentration (41).

Platelet aggregation could not be induced by $r$ 116(P503L) in the absence of ristocetin, even when used at a 10-fold higher concentration than that effective in the presence of $0.15 \mathrm{mg} /$ $\mathrm{ml}$ ristocetin. This is in apparent agreement with the observation that platelet aggregation does not occur in patients with the Malmö variant neither spontaneously nor after DDAVP administration (19). However, it has been reported in preliminary form that purified plasma vWF from one individual with this variant ( patient 2 of this study) can induce ristocetin-independent platelet aggregation when used at concentrations, between 30 and $80 \mu \mathrm{g} / \mathrm{ml}(42)$, that may not be attained in vivo even after DDAVP. This finding supports the concept that the 
Pro $^{503} \rightarrow$ Leu mutation results in increased vWF affinity for platelets even in the absence of ristocetin, although such an increase is clearly less pronounced than that caused by type IIB mutations. The latter statement is justified by the lower amounts of purified type IIB vWF necessary to induce aggregation $(43,44)$ as compared to type Malmö vWF (42). Thus, the phenotype of the von Willebrand disease variants type IIB and type Malmö (and presumably type I New York) is similar with regard to increased responsiveness to ristocetin and to the ability of vWF to induce platelet aggregation directly; rather, the difference appears to be in the extent to which the affinity for GP Ib is changed by different mutations in the GP Ib-binding domain of the molecule. This may be responsible for the other distinctive feature of type IIB patients, i.e. the disappearance of larger vWF multimers from the circulation $(7,43,44)$, due to their greater binding to platelet membranes at the vWF concentrations present in blood.

The failure to demonstrate a direct effect of the isolated GP Ib-binding domain on platelets may be due to inherent differences between multimeric vWF and dimeric $r 116$ fragment with regard to their respective ability to support platelet aggregation in the absence of ristocetin (27). Indeed, the dimeric fragment has only two GP Ib-binding sites per molecule, as opposed to several in a larger vWF multimer, and this may not be sufficient to support the aggregation (or agglutination) of platelets. Ristocetin may change this because it can dimerize in solution, it flocculates proteins and can bind to both the GP Ib-binding domain of $\mathrm{vWF}$ and to the platelet membrane (41), possibly creating functional "aggregates" of the dimeric fragment. It should be noted that, in unpublished studies, we found that a $r 116$ fragment containing a type IIB mutation also failed to induce platelet aggregation directly, even though the same fragment could clearly bind to GP Ib in the absence of any modulator (3). In this regard, then, expression of the isolated GP Ib-binding domain cannot reproduce a functional characteristic of the mutant multimeric vWF.

The exact structure and functional conformation of the GP Ib-binding site in the vWF subunit have yet to be clarified, although candidate regions have been identified as possible receptor recognition sites $(32,45)$. Moreover, evidence from several studies suggests that the disulfide loop between $\mathrm{Cys}^{509}$ and Cys $^{695}$ can modulate the affinity of vWF for GP Ib. For example, botrocetin, a snake venom protein, forms a bimolecular complex with vWF that then binds to GP Ib with high affinity; botrocetin has been shown to bind to three different segments of sequence within the above mentioned disulfide loop (46). In type IIB von Willebrand disease, characterized by increased vWF affinity for GP Ib $(5,6)$, all the reported mutations considered to be responsible for the functional abnormality are within the same disulfide loop; moreover, six of them are in the first botrocetin-binding segment and two in the second one $(3,11-$ $13,46)$. Porcine vWF, which spontaneously aggregates human platelets, displays interesting differences as compared to human vWF in the region corresponding to the first botrocetinbinding segment: in fact, it contains three amino acid substitutions (47) corresponding to those found in human type IIB vWF, which also aggregates normal human platelets $(43,44)$. All these results support the contention that conformational transitions within the disulfide loop between Cys ${ }^{509}$ and Cys ${ }^{695}$ regulate the affinity of vWF for GP Ib. We have now found that an amino acid change outside of the 509-695 disulfide loop in the Al domain of $\mathrm{vWF}$ can also lead to heightened interaction with GP Ib, suggesting that the latter event is regulated by an extended region in the GP Ib-binding domain of vWF with the contribution of previously unidentified residues. The fact that some of the patients reported here had negligible bleeding symptoms (patients 1 and 3 ) raises the intriguing possibility that other vWF mutations affecting the affinity for GP Ib may be found in the normal population.

The origin of the mutations described here is presently a matter of speculation, but it is clear from the characterization of different polymorphic markers of the vWF gene that they have occurred independently in the three families studied. Since the base changes found in the patients are $\mathrm{C} \rightarrow \mathrm{T}$ transitions at $\mathrm{CpG}$ dinucleotides in either the coding or non-coding strands, the codons for residues 500 and 503 in mature vWF may represent preferential sites of point mutations that tend to occur together for reasons that remain to be clarified. Nevertheless, since the single point mutation in the codon for residue 503 has arisen as an isolated event in the family of patient 1 , this explanation is not of general validity. Alternatively, if the mutation in the codon for residue 500 were a common polymorphism, it would be possible to argue for the fortuitous association between three independent mutational events in the codon for residue 503 and one of the two polymorphic sequences at position 500. Against this possibility is the fact that, in a preliminary survey ( 53 individuals) of the normal population, the mutation at position 500 was never identified, thus making its casual association with the Pro ${ }^{503} \rightarrow$ Leu mutation unlikely. Another intriguing possibility is suggested by the observation that, in two of the families studied (patients 2 and 3 in one family and patient 4 in the other), the two linked mutations create a gene sequence identical to that found in the corresponding segment of the vWF pseudogene; thus, they may have been the result of gene conversion, i.e., a nonreciprocal recombination event in which a segment of one gene replaces the corresponding segment of a related gene (48). Such a mechanism has been implicated as the cause of human steroid 21 -hydroxylase deficiency $(49,50)$. Although in the latter situation the gene and pseudogene involved are located on the same chromosome, the occurrence of heterochromosomal gene conversion has been clearly documented in lower organisms (51) and cannot be excluded in humans (48). In the case considered here, the sequence of vWF pseudogene implicated in the conversion event would be no more than 99 basepairs, representing the size of DNA segment that includes the two mutations present in the patients and is delimited by two mutations present in the pseudogene (26) but none in the mutant alleles. It is interesting to speculate that, if the pseudogene has diverged from the vWF gene especially at mutational hot spots, there may be other von Willebrand disease variants caused by amino acid substitutions coded for by sequences identical to those in the vWF pseudogene. Such a possibility is presently under investigation.

\section{Acknowledgments}

We thank Prof. Inga Marie Nilsson and Dr. Erik Berntorp for help in obtaining samples from patient 1; and Dr. Akira Yoshioka of Nara Medical College, Nara, Japan, for providing the monoclonal antibody NMC-4.

This work was supported in part by grant HL-48728 from the National Institutes of Health. We acknowledge the Sam and Rose Stein Trust Fund in the Department of Molecular and Experimental Medi- 
cine at The Scripps Research Institute for financial support to the DNA core facility. Additional support for the General Clinical Research Center (National Institutes of Health grant RR0833) and from the Swedish Medical Research Council (grants 4997 and 9627) is also acknowledged.

This is publication 7499-MEM/CVB from The Scripps Research Institute.

Note added in proof. After submission of the manuscript for this article, Dr. Harvey Weiss provided us with blood from a member of the original family in which the type I New York variant of von Willebrand disease was identified (patient 3 in reference 18). We found that this individual was heterozygous for the same mutations of vWF codons 500 and 503 reported in this paper. Thus, "type I New York" and "type Malmö" von Willebrand disease are identical variants.

\section{References}

1. Holmberg, L., and I. M. Nilsson. 1992. von Willebrand's Disease. Eur. J. Haematol. 48:127-141.

2. Ruggeri, Z. M., and J. Ware. 1992. The structure and function of von Willebrand factor. Thromb. Haemostasis. 67:594-599.

3. Ware, J., J. A. Dent, H. Azuma, M. Sugimoto, P. A. Kyrle, A. Yoshioka, and Z. M. Ruggeri. 1991. Identification of a point mutation in Type IIB von Willebrand Disease illustrating the regulation of von Willebrand Factor affinity for the platelet GP Ib-IX receptor. Proc. Natl. Acad. Sci. USA. 88:2946-2950.

4. Ginsburg, D., B. A. Konkle, J. C. Gill, R. R. Montgomery, P. L. Bockenstedt, T. A. Johnson, and A. Y. Yang. 1989. Molecular basis of human von Willebrand disease: Analysis of platelet von Willebrand factor mRNA. Proc. Natl. Acad. Sci. USA. 86:3723-3727.

5. De Marco, L., M. Mazzucato, D. De Roia, A. Casonato, A. B. Federici, A Girolami, and Z. M. Ruggeri. 1990. Distinct abnormalities in the interaction of purified IIA and IIB von Willebrand factor with the two platelet binding sites GP Ib-IX and GP IIb-IIIa. J. Clin. Invest. 86:785-792.

6. Ruggeri, Z. M., F. I. Pareti, P. M. Mannucci, N. Ciavarella, and T. S. Zimmerman. 1980. Heightened interaction between platelets and Factor VIII/ von Willebrand factor in a new subtype of von Willebrand's disease. $N$. Engl. J. Med. 302:1047-1051.

7. Ruggeri, Z. M., and T. S. Zimmerman. 1980. Variant von Willebrand's disease: characterization of two subtypes by analysis of multimeric composition of factor VIII/von Willebrand factor in plasma and platelets. J. Clin. Invest. 65:1318-1325.

8. Gralnick, H. R., S. B. Williams, L. P. McKeown, M. E. Rick, P. Maisonneuve, C. Jenneau, and Y. Sultan. 1985. Von Willebrand's disease with spontaneous platelet aggregation induced by an abnormal plasma von Willebrand factor. J. Clin. Invest. 76:1522-1529.

9. Saba, H. I., S. R. Saba, J. Dent, Z. M. Ruggeri, and T. S. Zimmerman. 1985 Type IIB Tampa: A variant of von Willebrand disease with chronic thrombocytopenia, circulating platelet aggregates, and spontaneous platelet aggregation. Blood. 66:282-286.

10. Holmberg, L., I. M. Nilsson, L. Borge, M. Gunnarsson, and E. Sjorin. 1983. Platelet aggregation induced by 1-desamino-8-D-arginine vasopressin (DDAVP) in Type IIB von Willebrand's disease. N. Engl. J. Med. 309:816-821

11. Cooney, K. A., W. C. Nichols, M. E. Bruck, W. F. Bahou, A. D. Shapiro, E. J. W. Bowie, H. R. Gralnick, and D. Ginsburg. 1991. The molecular defect in type IIB von Willebrand disease: identification of four potential missense mutations within the putative GPIb binding domain. J. Clin. Invest. 87:1227-1233.

12. Randi, A. M., I. Rabinowitz, D. J. Mancuso, P. M. Mannucci, and J. E. Sadler. 1991. Molecular basis of von Willebrand disease type IIB: candidate mutations cluster in one disulfide loop between proposed platelet glycoproteins $\mathrm{Ib}$ binding sequences. J. Clin. Invest. 87:1220-1226.

13. Ribba, A. S. J. M. Lavergne, B. R. Bahnak, A. Derlon, G. Pietu, and D. Meyer. 1991. Duplication of a methionine within the glycoprotein Ib binding domain of von Willebrand factor detected by denaturing gradient gel electrophoresis in a patient with type IIB von Willebrand disease. Blood. 78:1738-1743.

14. Miller, J. L., and A. Castella. 1982. Platelet-type von Willebrand's disease: characterization of a new bleeding disorder. Blood. 60:790-794.

15. Miller, J. L., J. M. Kupinski, A. Castella, and Z. M. Ruggeri. 1983. von Willebrand factor binds to platelets and induces aggregation in platelet-type but not type IIB von Willebrand disease. J. Clin. Invest. 72:1532-1542.

16. Weiss, H. J., D. Meyer, R. Rabinowitz, G. Pietu, J. Girma, W. J. Vicic, and J. Rogers. 1982. Pseudo von Willebrand's disease: an intrinsic platelet defect with aggregation by unmodified human factor VIII/von Willebrand factor and enhanced adsorption of its high molecular weight multimers. N. Engl. J. Med. 306:326-333.

17. Miller, J. L., D. Cunningham, V. A. Lyle, and C. L. Finch. 1991. Mutation in the gene encoding the $\alpha$ chain of platelet glycoprotein Ib in platelet-type von Willebrand disease. Proc. Natl. Acad. Sci. USA. 88:4761-4765.

18. Weiss, H. J. and I. I. Sussman. 1986. A new von Willebrand variant (type I, New York): increased ristocetin-induced platelet aggregation and plasma von Willebrand factor containing the full range of multimers. Blood. 68:149-156.

19. Holmberg, L., E. Berntorp, M. Donner, and I. M. Nilsson. 1986. von Willebrand's disease characterized by increased ristocetin sensitivity and the presence of all von Willebrand factor multimers in plasma. Blood. 68:668-672.

20. Ruggeri, Z. M., and T. S. Zimmerman. 1981. The complex multimeric composition of Factor VIII/von Willebrand factor. Blood. 57:1140-1143.

21. Barbui, T., F. Baudo, N. Ciavarella, G. Mariani, A. Capitanio, P. M. Mannucci, F. I. Pareti, Z. M. Ruggeri, and Italian Working Group. 1977. Spectrum of von Willebrand's disease: a study of 100 cases. Br. J. Haematol. 35:101112.

22. Blin, N., and D. W. Stafford. 1976. A general method for isolation of high molecular weight DNA from eukaryocytes. Nucleic Acids Res. 3:2303-2308.

23. Newman, P. J., J. Gorski, G. C. White, S. Gidwitz, C. J. Cretney, and R. H. Aster. 1988. Enzymatic amplification of platelet-specific messenger RNA using the polymerase chain reaction. J. Clin. Invest. 82:739-743.

24. Mancuso, D. J., E. A. Tuley, L. A. Westfield, N. K. Worrall, B. B. SheltonInloes, J. M. Sorace, Y. G. Alevy, and J. E. Sadler. 1989. Structure of the gene for human von Willebrand factor. J. Biol. Chem. 264:19514-19527.

25. Saiki, R. K., D. H. Gelfand, S. Stoffel, S. J. Scharf, R. Higuchi, G. T. Horn, K. B. Mullis, and H. A. Erlich. 1988. Primer-directed enzymatic amplification of DNA with a thermostable DNA polymerase. Science (Wash. DC). 239:487-491.

26. Mancuso, D. J., E. A. Tuley, L. A. Westfield, T. L. Lester-Mancuso, M. M Le Beau, J. M. Sorace, and J. E. Sadler. 1991. Human von Willebrand factor gene and pseudogene: structural analysis and differentiation by polymerase chain reaction. Biochemistry. 30:253-269.

27. Azuma, H., J. A. Dent, M. Sugimoto, Z. M. Ruggeri, and J. Ware. 1991. Independent assembly and secretion of a dimeric adhesive domain of von Willebrand factor containing the glycoprotein Ib-binding site. J. Biol. Chem. 266:12342-12347.

28. Kunkel, T. A., J. D. Roberts, and R. A. Zakour. 1987. Rapid and efficient site-specific mutagenesis without phenotypic selection. Methods Enzymol. 154:367-383.

29. Chen, C., and H. Okayama. 1987. High-efficiency transformation of mammalian cells by plasmid DNA. Mol. Cell. Biol. 7:2745-2752.

30. Laurell, C. B. 1966. Quantitative estimation of proteins by electrophoresis in agarose gel containing antibodies. Anal. Biochem. 15:45-52.

31. Zimmerman, T. S., O. D. Ratnoff, and A. S. Littell. 1971. Detection of carriers of classic hemophilia using an immunologic assay for antihemophilic factor (Factor VIII). J. Clin. Invest. 50:255-258.

32. Mohri, H., Y. Fujimura, M. Shima, A. Yoshioka, R. A. Houghten, Z. M Ruggeri, and T. S. Zimmerman. 1988. Structure of the von Willebrand factor domain interacting with glycoprotein Ib. J. Biol. Chem. 263:17901-17904.

33. Fujimura, Y., Y. Usami, K. Titani, K. Niinomi, K. Nishio, T. Takase, A. Yoshioka, and H. Fukui. 1991. Studies on anti-von Willebrand factor (vWF) monoclonal antibody NMC-4, which inhibits both ristocetin- and botrocetin-induced vWF binding to platelet glycoprotein Ib. Blood. 77:113-120.

34. Walsh, P. N., D. C. B. Mills, and J. G. White. 1977. Metabolism and function of human platelets washed by albumin density gradient separation. $B r$. J. Haematol. 36:281-298.

35. Ruggeri, Z. M., L. De Marco, L. Gatti, R. Bader, and R. R. Montgomery. 1983. Platelets have more than one binding site for von Willebrand factor. $J$. Clin. Invest. 72:1-12.

36. Donner, M., L. Holmberg, A. C. Kristoffersson, and I. M. Nilsson. 1991. An HphI polymorphism in exon 28 of the von Willebrand factor gene, and its frequency among patients with various forms of von Willebrand's disease. $B r . J$. Haematol. 78:403-407.

37. Weiss, H. J., V. T. Turitto, and H. R. Baumgartner. 1978. Effect of shear rate on platelet interaction with subendothelium in citrated and native blood. I Shear rate-dependent decrease of adhesion in von Willebrand's disease and the Bernard-Soulier syndrome. J. Lab. Clin. Med. 92:750-764.

38. Ikeda, Y., M. Handa, K. Kawano, T. Kamata, M. Murata, Y. Araki, H Anbo, Y. Kawai, K. Watanabe, I. Itagaki, et al. 1991. The role of von Willebrand Factor and fibrinogen in platelet aggregation under varying shear stress. J. Clin. Invest. 87:1234-1240.

39. Fujimura, Y., K. Titani, L. Z. Holland, S. R. Russell, J. R. Roberts, J. H. Elder, Z. M. Ruggeri, and T. S. Zimmerman. 1986. von Willebrand factor. A reduced and alkylated $52 / 48 \mathrm{kDa}$ fragment beginning at amino acid residue 449 contains the domain interacting with platelet glycoprotein Ib. J. Biol. Chem. 261:381-385.

40. Mohri, H., A. Yoshioka, T. S. Zimmerman, and Z. M. Ruggeri. 1989 Isolation of the von Willebrand factor domain interacting with platelet glycoprotein Ib, heparin, and collagen, and characterization of its three distinct functional sites. J. Biol. Chem. 264:17361-17367.

41. Scott, J. P., R. R. Montgomery, and G. S. Retzinger. 1991. Dimeric ristocetin flocculates proteins, binds to platelets, and mediates von Willebrand factor-dependent agglutination of platelets. J. Biol. Chem. 266:8149-8155. 
42. De Marco, L., M. Mazzucato, U. Budde, P. Pradella, A. Masotti, and Z. M. Ruggeri. 1991. Purification and characterization of the platelet aggregating properties of type I New York von Willebrand factor (NY-vWF). Blood. 78:149a (Abstr.)

43. De Marco, L., A. Girolami, T. S. Zimmerman, and Z. M. Ruggeri. 1985. Interaction of purified IIB von Willebrand factor with the platelet membrane glycoprotein Ib induces fibrinogen binding to the glycoprotein IIb/IIla complex and initiates aggregation. Proc. Natl. Acad. Sci. USA. 82:7424-7428.

44. De Marco, L., M. Mazzucato, M. G. Del Ben, U. Budde, A. B. Federici, A. Girolami, and Z. M. Ruggeri. 1987. Type IIB von Willebrand factor with normal sialic acid content induces platelet aggregation in the absence of ristocetin. Role of platelet activation, fibrinogen, and two distinct membrane receptors. J. Clin. Invest. 80:475-482.

45. Berndt, M. C., C. M. Ward, W. J. Booth, P. A. Castaldi, A. V. Mazurov, and R. K. Andrews. 1992. Identification of aspartic acid 514 through glutamic acid 542 as a glycoprotein Ib-IX complex receptor recognition sequence in von Willebrand factor: mechanism of modulation of von Willebrand factor by ristocetin and botrocetin. Biochemistry. 31:11144-11151.

46. Sugimoto, M., H. Mohri, R. A. McClintock, and Z. M. Ruggeri. 1991. Identification of discontinuous von Willebrand factor sequences involved in complex formation with botrocetin: a model for the regulation of von Willebrand factor binding to platelet glycoprotein Ib. J. Biol. Chem. 266:18172-18178.

47. Bahnak, B. R., J-M. Lavergne, V. Ferreira, D. Kerbiriou-Nabias, and D.
Meyer. 1992. Comparison of the primary structure of the functional domains of human and porcine von Willebrand factor that mediate platelet adhesion. Biochem. Biophys. Res. Commun. 182:561-568.

48. Baltimore, D. 1981. Gene conversion: some implications for immunoglobulin genes. Cell. 24:592-594.

49. Higashi, Y., A. Tanae, H. Inoue, and Y. Fujii-Kuriyama. 1988. Evidence for frequent gene conversion in the steroid 21 -hydroxylase P-450(C21) gene: implications for steroid 21-hydroxylase deficiency. Am. J. Hum. Genet. 42:1725.

50. Urabe, K., A. Kimura, F. Harada, T. Iwanaga, and T. Sasazuki. 1990. Gene conversion in steroid 21-hydroxylase genes. Am. J. Hum. Genet. 46:11781186.

51. Petes, T. D., and C. W. Hill. 1988. Recombination between repeated genes in microorganisms. Annu. Rev. Genet. 22:147-168.

52. Bernardi, F., G. Marchetti, V. Bertagnolo, L. Faggioli, and L. del Senno. 1987. Two TaqI RFLPs in the human von Willebrand gene. Nucleic Acids Res. 15:1347.

53. Quadt, R., C. L. Verweij, C. J. M. de Vries, E. Briët, and H. Pannekoek. 1986. A polymorphic Xba I site within the human von Willebrand factor (vWF) gene identified by a vWF cDNA clone. Nucleic Acids Res. 14:7139.

54. Peake, I. R., D. Bowen, P. Bignell, M. B. Liddell, J. E. Sadler, G. Standen, and A. L. Bloom. 1990. Family studies and prenatal diagnosis in severe von Willebrand disease by polymerase chain reaction amplification of a variable number tandem repeat region of the von Willebrand factor gene. Blood. 76:555-561. 